\title{
Interaction of a Moreton/EIT wave and a coronal hole
}

\author{
Astrid M. Veronig \\ IGAM/Institute of Physics, University of Graz, Universitätsplatz 5, A-8010 Graz, Austria; \\ asv@igam.uni-graz.at \\ Manuela Temmer and Bojan Vršnak \\ Hvar Observatory, Faculty of Geodesy, Kačićeva 26, HR-10000 Zagreb, Croatia; \\ manuela.temmer@uni-graz.at,bvrsnak@geodet.geof.hr \\ and \\ Julia K. Thalmann \\ IGAM/Institute of Physics, University of Graz, Universitätsplatz 5, A-8010 Graz, Austria; \\ jut@igam.uni-graz.at
}

\begin{abstract}
We report high-cadence $\mathrm{H} \alpha$ observations of a distinct Moreton wave observed at Kanzelhöhe Solar Observatory associated with the 3B/X3.8 flare and CME event of 2005 January 17. The Moreton wave can be identified in about $40 \mathrm{H} \alpha$ frames over a period of $7 \mathrm{~min}$. The EIT wave is observed in only one frame but the derived propagation distance is close to that of the simultaneously measured Moreton wave fronts indicating that they are closely associated phenomena. The large angular extent of the Moreton wave allows us to study the wave kinematics in different propagation directions with respect to the location of a polar coronal hole $(\mathrm{CH})$. In particular we find that the wave segment whose propagation direction is perpendicular to the $\mathrm{CH}$ boundary ("frontal encounter") is stopped by the $\mathrm{CH}$ which is in accordance with observations reported from EIT waves (Thompson et al. 1998). However, we also find that at a tongueshaped edge of the coronal hole, where the front orientation is perpendicular to the $\mathrm{CH}$ boundary (the wave "slides along" the boundary), the wave signatures can be found up to $100 \mathrm{Mm}$ inside the $\mathrm{CH}$. These findings are briefly discussed in the frame of recent modeling results.
\end{abstract}

Subject headings: shock waves — Sun: corona — Sun: flares

\section{Introduction}

Moreton waves are arc-like propagating disturbances observed in chromospheric $\mathrm{H} \alpha$ images in association with solar flare/CME events (Moreton \& Ramsey 1960; Athay \& Moreton 1961). Uchida 
(1968) developed the theory that the Moreton waves are just the surface track of a fast-mode MHD wave which is coronal in nature. When the coronal wave front sweeps over the chromosphere, the enhanced pressure behind the wave front compresses the plasma which is observed as the typical down-up swing in $\mathrm{H} \alpha$ filtergrams. In recent years, the Extreme-ultraviolet Imaging Telescope (EIT) onboard the Solar and Heliospheric Observatory (SoHO) directly imaged many globally propagating disturbances in the low corona (e.g. Thompson et al. 1998, 1999), so-called EIT waves. Soon thereafter, coronal waves were also imaged in soft X-rays by the SXT/Yohkoh (e.g. Khan \& Aurass 2002; Hudson et al. 2003) and SXI/GOES-12 (Warmuth et al. 2005; Vršnak et al. 2006) instruments. Whether the EIT (and soft X-ray) waves are the coronal counterpart of Moreton waves as anticipated by Uchida's model is still a matter of debate (e.g. Vršnak 2005; Chen et al. 2005), in particular as EIT waves are on average a factor 2-3 slower than Moreton waves (Klassen et al. 2000; for a discussion on statistical selection effects see Warmuth et al. 2001). However, in cases where both the chromospheric Moreton and the coronal EIT wave were observed, they were lying on closely related kinematical curves providing strong evidence that, at least in these events, the Moreton waves are indeed the chromospheric counterpart of the coronal EIT waves (Thompson et al. 1999; Warmuth et al. 2001; Biesecker et al. 2002; Vršnak et al. 2002; Gilbert et al. 2004; Warmuth et al. 2004; Vršnak et al. 2006).

Some observations show that EIT waves tend to avoid active regions (ARs; Thompson et al. 1999; Wills-Davey \& Thompson 1999) and stop at the boundaries of coronal holes (CHs; Thompson et al. 1998) as well as near the separatrix between ARs where they may appear as a "stationary" front (Delannée \& Aulanier 1999). These observations were reproduced in numerical simulations treating EIT waves as fast-mode MHD waves (Wang 2000; Wu et al. 2001; Ofman \& Thompson 2002) showing that the wave undergoes strong refraction and deflection when interacting with ARs and CHs, which are both high Alfvén velocity regions compared to the quiet Sun. Delannée \& Aulanier (1999) and Chen et al. (2002, 2005) proposed different models where EIT waves are not real waves but the signature of a propagating perturbation related to magnetic field line opening in the wake of the associated CME.

Observational studies of the interaction of coronal waves with ARs and CHs are rare and usually hampered by the low cadence of the EIT instrument ( 12-15 min), which moreover restricts the studies to slow waves. In this paper we report high cadence $\mathrm{H} \alpha$ observations of a fast Moreton wave with well defined fronts extending over a large angular sector of $\sim 130^{\circ}$ which can be followed up to $\sim 500 \mathrm{Mm}$ from the wave ignition center, i.e. considerably farther out than Moreton waves are usually observed. Due to the 12-min cadence, the EIT wave can be observed solely in one single frame but is roughly co-spatial with the simultaneously observed Moreton wave fronts as expected from Uchida's theory. The high-cadence $\mathrm{H} \alpha$ observations of this distinct Moreton wave provide us with the possibility to study in detail the interaction of the coronal wave as traced by its chromospheric ground track and a polar $\mathrm{CH}$, which cannot be directly accessed in such detail with present coronal imagers. 


\section{Data and Observations}

The Moreton wave associated with the 3B/X3.8 flare of 2005 January 17 (N15,W25; peak time $\sim 09: 50 \mathrm{UT}$ ) was observed with high time cadence in full-disk $\mathrm{H} \alpha$ filtergrams at the Kanzelhöhe Solar Observatory (KSO; Otruba \& Pötzi 2003). KSO routinely takes full-disk H $\alpha$ images with a cadence of $\sim 5-10 \mathrm{~s}$ and a spatial resolution of $2.2 /$ pixel. When the flare-mode is triggered, additionally images in the red and blue wing of the $\mathrm{H} \alpha$ spectral line (at the off-band center wavelengths of $\mathrm{H} \alpha+0.4 \AA$ and $\mathrm{H} \alpha-0.3 \AA$, respectively) are taken with a cadence of $\sim 60-80 \mathrm{~s}$ in each wing.

The flare evolution is complex and consists of several stages. The GOES flux (Fig. 1a) shows a sudden increase to M2 level around 8:00 UT, then gradually (over 100 min) increases further to the X2 level, and finally shows an impulsive enhancement at 09:40 UT which reaches the X4 peak at 09:50 UT. The Moreton wave is associated with this last peak. For this phase, also hard X-ray data from the Reuven Ramaty High Energy Solar Spectroscopic Imager (RHESSI; Lin et al. 2002) are available. Between about 09:41 and 09:58 UT spiky emission at high X-ray energies is observed (Figure 1b).

We also checked images from the Extreme-ultraviolet Imaging Telescope (EIT; Delaboudinière et al. 1995) for wave signatures and the location of the $\mathrm{CH}$ boundaries. Figure 2 shows an EIT Fe XII $195 \AA$ (peak formation temperature of 1.6 MK) preflare image of the northern hemisphere of the Sun. The boundary of the polar $\mathrm{CH}$, which is in the direction of propagation of the Moreton/EIT wave, is outlined on the image. Since the exact $\mathrm{CH}$ boundary is difficult to determine, we draw both the outer edge as well as an estimate of the diffuse inner edge of the $\mathrm{CH}$ boundary.

\section{Results}

In total, the Moreton wave fronts could be identified in $\sim 30$ images taken in the center, 6 images acquired in the red and 5 images in the blue wing of the $\mathrm{H} \alpha$ spectral line during the period 09:43:25 to 09:50:38 UT. Figure 3 shows a sequence of $\mathrm{H} \alpha+0.4 \AA$ running difference images of the Moreton wave. In Figure 4, the leading edges of all visually determined wave fronts are drawn on an $\mathrm{H} \alpha$ flare image. The boundaries of the polar $\mathrm{CH}$ (inner and outer estimates) determined from the EIT $195 \AA$ image shown in Fig. 2 are also indicated in Figs. 3 and 4 .

The Moreton wave fronts extend across a maximum sector of $\sim 130^{\circ}$. When approaching the $\mathrm{CH}$, there is a distinct change in the wave propagation characteristics leading to a distortion of the circular shape of the wavefront which can be directly seen, e.g., in Figs. 3d,e. From Figure 4 it is evident that the Moreton wave fronts partially intrude into the $\mathrm{CH}$. The penetration depth depends on the estimated boundaries of the $\mathrm{CH}$. However, even for the inner estimate, some wave fronts are observed inside the $\mathrm{CH}$. Further evidence that the wave was able to intrude into the $\mathrm{CH}$ is provided by a feature located inside the $\mathrm{CH}$ (marked as "B" in Fig. 4) that was activated at the time when the wave passed its position. The wave also activated another feature ("A") that is 
located along the border of the $\mathrm{CH}$.

Figure 5 shows two EIT running difference images. Due to the low cadence of EIT ( $\sim 12 \mathrm{~min})$, there is only one EIT wavefront visible on-disk at 09:46:55 UT (left panel). In the next EIT frame, the wave has already passed the disk and caused a coronal dimming behind it (right panel). The figure also reveals that a bright elongated feature located along the $\mathrm{CH}$ boundary (outer estimate) disappeared after the wave passage. Since this feature reappeared in later images ( $\gtrsim 12 \mathrm{UT})$, it is possible that it was compressionally heated when hit by the wave, and was thus no longer visible in the EIT $195 \AA$ passband. From the EIT observations we find no indication that the wave intruded into the CH. This may be related to the low image cadence of EIT which does not allow us to get insight into the EIT wave kinematics, or it may be a real difference between the EIT and $\mathrm{H} \alpha$ Moreton wave propagation characteristics.

The Moreton wave ignition site was estimated by applying circular fits to the earliest observed $\mathrm{H} \alpha$ wave fronts, whereby the projection effect due to the spherical solar surface was taken into account (see Warmuth et al. 2004). We found the ignition site at $x \approx 505^{\prime \prime}$ and $y \approx 300^{\prime \prime}$ from Sun center, which is approximately $50^{\prime \prime} \mathrm{NW}$ from the centroid of the flare area impulsively activated during this time and outside the strong sunspots of the active region (cf. Fig 4). We then determined for each point of the wave front, i.e. considering its total angular extent, its distance from the wave ignition site measured along great circles on the solar surface. The derived time-distance diagram is plotted in Fig. 6a. At each instant the mean value derived from the distances of all points on one wave front together with the standard deviation (error bars) is plotted. The first wave front observed in $\mathrm{H} \alpha+0.4 \AA$ at 09:43:25 UT was ignored in the kinematical curves since it was very diffuse and therefore difficult to measure. In $\mathrm{H} \alpha$, the wave propagation can be observed up to a distance of $\sim 500 \mathrm{Mm}$ from the ignition site. This is considerably farther out than Moreton waves are usually observed. Warmuth et al. (2004) report an average value for the maximum distance of $300 \mathrm{Mm}$.

Also plotted in Fig. 6a are the results from a linear and quadratic least squares fit to the data. From the linear fit we obtain a mean wave speed of $930 \pm 30 \mathrm{~km} \mathrm{~s}^{-1}$. The quadratic fit gives a significant deceleration of $-1240 \pm 470 \mathrm{~m} \mathrm{~s}^{-2}$. From the quadratic fit we find a back-extrapolated start time of the wave of 09:42:40 UT $\pm 30 \mathrm{~s}$, from the linear fit it is 09:41:50 UT $\pm 20 \mathrm{~s}$. These start times point to the impulsive onset of the flare hard X-ray emission observed by RHESSI (see the first peak at 50-100 keV in Fig. 1). The distance derived from the EIT wave front at 09:46:55 UT indicates that the EIT wave is $\sim 50 \mathrm{Mm}$ ahead but roughly co-spatial with the instantaneously observed Moreton wave fronts. This finding is in contrast to the statistical result that EIT waves are significantly (by a factor 2-3) slower than Moreton waves (Klassen et al. 2000) but agrees with Uchida's sweeping skirt hypothesis in which the $\mathrm{H} \alpha$ Moreton wave is the chromospheric surface track of the coronal wave as observed by the EIT, SXT and SXI instruments.

In Fig. 6b, we present the time-distance diagram separately for three propagation sectors. Sector 1 includes the wave segment where the wave front and $\mathrm{CH}$ boundary are quasi parallel 
("frontal encounter" of the wave). It is defined by the angular sector $\left[90^{\circ}, 110^{\circ}\right]$ measured from the $x$-axis with the wave center as origin. Sector 2 covers the direction of the western boundary of the $\mathrm{CH}$ (revealing a "tongue"-like shape) where the wave fronts impinge quasi-parallel as well as normal to the $\mathrm{CH}$ boundaries (defined by $\left[65^{\circ}, 90^{\circ}\right]$ ). Sector 3 is in the direction that is unaffected by any $\mathrm{CH}$ boundary (defined by $\left[-20^{\circ}, 65^{\circ}\right]$ ). The three sectors are indicated in Fig. 4 .

Up to about 09:46 UT all three kinematical curves are very similar. Thereafter, the wave segments of sectors 1 and 2 (i.e. in direction of the $\mathrm{CH}$ ) slow down with respect to the undisturbed direction (sector 3). At 09:48:20 UT the wave segments in sector 1 stop at a distance of about $300 \mathrm{Mm}$, i.e. closely ahead of the $\mathrm{CH}$ boundary (see also Fig. 4). The wave propagating into the direction of the $\mathrm{CH}$ tongue (sector 2) also shows deceleration when approaching the $\mathrm{CH}$, but some of the wavefronts intrude into the $\mathrm{CH}$. At that time their propagation characteristics (inside the $\mathrm{CH}$ ) are different from that of the wave segments propagating into sector 3, i.e. the undisturbed direction. In particular, their distance to the wave ignition center is about $60-90 \mathrm{Mm}$ smaller than that of the wave front segments not disturbed by the $\mathrm{CH}$, indicating that the wave fronts are significantly distorted by the presence of the $\mathrm{CH}$.

\section{Discussion and Conclusions}

High cadence $\mathrm{H} \alpha$ observations of a distinct Moreton wave extending over an angular extent of $\sim 130^{\circ}$ allowed us to study the propagation characteristics in different directions with regard to the presence of a polar $\mathrm{CH}$. In particular we find that in the frontal encounter (sector 1), the wave stops at the $\mathrm{CH}$ boundary. On the other hand, at the western side of the $\mathrm{CH}$ where the front orientation is normal to the $\mathrm{CH}$ boundary, the wave can to a certain degree intrude into the $\mathrm{CH}$. Depending on the different (i.e. inner/outer) $\mathrm{CH}$ boundary estimates we find a maximum penetration depth of 65 and $100 \mathrm{Mm}$, respectively. From the EIT observations we find no indication that the wave was able to intrude into the $\mathrm{CH}$. This may be related to the low image cadence of EIT - while the Moreton wave can be measured in 40 frames, the EIT front is observed only in a single frame - or it may be a real difference between the EIT and $\mathrm{H} \alpha$ wave propagation characteristics.

In the MHD fast-mode hypothesis, the velocity of the coronal wave which propagates basically perpendicular to the magnetic field lines, is determined by the fast magnetosonic speed $v_{\mathrm{ms}}=$ $\left(v_{A}^{2}+c_{s}^{2}\right)^{1 / 2}$ with $v_{A}=B /(4 \pi \rho)^{1 / 2}$ the Alfvén speed and $c_{s}=\left(\gamma k T / \bar{\mu} m_{u}\right)^{1 / 2}$ the sonic speed, where $B$ denotes the magnetic field strength, $\rho$ the mass density, $n$ the particle density, $T$ the temperature, $\gamma$ the adiabatic exponent, $k$ the Boltzmann constant, $m_{u}$ the atomic mass unit, and $\bar{\mu}$ the mean molecular mass. The Moreton wave of 17 January 2005 has its starting point at the NW border of AR 10720 and propagates through quiet Sun regions, partly into the direction of the northern polar $\mathrm{CH}$. The higher magnetosonic speed in CHs compared to quiet Sun regions, which causes refraction and reflection of the wave at the $\mathrm{CH}$, is mainly due to the higher Alfvén velocity in CHs. The density in CHs is about a factor 3-10 smaller compared to the quiet Sun (Gabriel 1992; Young

\& Esser 1999; Cranmer 2000). Using the solar minimum magnetic field model by Banaszkiewicz 
et al. (1998) which is a reasonable representation of the large scale magnetic field outside ARs, we find that the global field is about a factor 3 smaller at the latitude of the wave initiation $\left(\sim 15^{\circ}\right)$ than at $\sim 35-40^{\circ}$, where the southern boundary of the $\mathrm{CH}$ is located. The temperature in $\mathrm{CHs}$ is up to a factor 2 smaller than the ambient coronal plasma. Thus, the Alfvén velocity in CHs is on average a factor 5-9 higher than in the ambient quiet Sun coronal plasma, whereas the sonic speed is about a factor 1.4 smaller. Assuming that in the corona the Alfvén velocity is significantly higher than the sonic speed (low- $\beta$ plasma), this implies that the fast magnetosonic speed in the $\mathrm{CH}$ is about 5-9 times higher than in the quiet corona. As argued by some authors (e.g. Wang 2000), in quiet regions the plasma- $\beta$ could be of the order of unity and thus the sonic velocity comparable to the Alfvén velocity. However, also in this case the fast magnetosonic speed in the CHs is still considerably higher than in quiet regions, namely by a factor of 3-6.

The stopping of the wave at the $\mathrm{CH}$ boundary in the case of the "frontal encounter" was numerically simulated by Wang (2000) and Wu et al. (2001). As the wave approaches a region of high magnetosonic speed it is refracted away from that region and the wave propagation is halted (Wang 2000; Wu et al. 2001). Due to the low values of the plasma-to-magnetic pressure ratio in the $\mathrm{CH}$ region, the mass motion is constrained (Wu et al. 2001) and the plasma piles up when the wave encounters the $\mathrm{CH}$ boundary. That could explain the activated (neutral line) feature "A". There could be also a small transmitted component of the waves as seen in the simulations by Ofman \& Thompson (2002). However, the magnitude of the transmitted component must be much smaller than that of the reflected component, and is therefore difficult to detect.

A related phenomenon is the leakage of a fast mode wave into the $\mathrm{CH}$ region (Wu et al. 2001). Our observations apparently reveal such a leakage at the western edge of the $\mathrm{CH}$, where the wave passed the $\mathrm{CH}$ tongue. After passing the tongue we still see wave signatures within the $\mathrm{CH}$ region. Moreover, the wave caused there an activation of feature "B". Since that happened in a different geometry (the wave is here "sliding along" the $\mathrm{CH}$ boundary), the activation was most likely due to the interaction of the wave flank that managed to penetrate into the $\mathrm{CH}$ from aside, and the preexisting magnetic structure therein. Such a process is possibly similar to the wave/AR interaction modeled by Ofman \& Thompson (2002), since ARs are as CHs high Alfvén/magnetosonic velocity regions. It was shown by Ofman \& Thompson (2002) that when the fast magnetosonic wave encounters an AR it undergoes strong reflection and refraction. On the other hand, transient currents are induced within the AR, and the resulting Lorentz force causes dynamical distortion of the magnetic field structure. The resulting magnetic compression of plasma generates flows which could be seen as an AR activation, similar to what we observed by the activation of feature B inside the $\mathrm{CH}$.

The present analysis shows the importance of high time cadence observations to interpret properly the phenomenon of the interaction of a coronal/Moreton wave with a $\mathrm{CH}$. Up to now, conclusions were drawn only from EIT observations which are taken with low time cadence, and thus might miss important details. 
We gratefully acknowledge support by the Austrian Fonds zur Förderung der wissenschaftlichen Forschung (FWF) under grants P15344 and J2512-N02. We thank the anonymous referee for the suggested improvements to the paper.

\section{REFERENCES}

Athay, R. G. \& Moreton, G. E. 1961, ApJ, 133, 935

Banaszkiewicz, M., Axford, W. I., \& McKenzie, J. F. 1998, A\&A, 337, 940

Biesecker, D. A., Myers, D. C., Thompson, B. J., Hammer, D. M., \& Vourlidas, A. 2002, ApJ, 569, 1009

Chen, P. F., Fang, C., \& Shibata, K. 2005, ApJ, 622, 1202

Chen, P. F., Wu, S. T., Shibata, K., \& Fang, C. 2002, ApJ, 572, L99

Cranmer, S. 2000, in Encyclopedia of Astronomy and Astrophysics, Ed. P. Murdin, 496-501

Delaboudinière, J.-P., Artzner, G. E., Brunaud, J. et al. 1995, Sol. Phys., 162, 291

Delannée, C. \& Aulanier, G. 1999, Sol. Phys., 190, 107

Gabriel, A. 1992, in The Sun: A Laboratory for Astrophysics, Eds. J. T. Schmelz and J. C. Brown, NATO ASI Series 373, 277-296

Gilbert, H. R., Holzer, T. E., Thompson, B. J., \& Burkepile, J. T. 2004, ApJ, 607, 540

Hudson, H. S., Khan, J. I., Lemen, J. R., Nitta, N. V., \& Uchida, Y. 2003, Sol. Phys., 212, 121

Khan, J. I. \& Aurass, H. 2002, A\&A, 383, 1018

Klassen, A., Aurass, H., Mann, G., \& Thompson, B. J. 2000, A\&AS, 141, 357

Lin, R. P., Dennis, B. R., Hurford, G. J. et al. 2002, Sol. Phys., 210, 3

Moreton, G. E. \& Ramsey, H. E. 1960, PASP, 72, 357

Ofman, L. \& Thompson, B. J. 2002, ApJ, 574, 440

Otruba, W. \& Pötzi, W. 2003, Hvar Observatory Bulletin, 27, 189

Thompson, B. J., Gurman, J. B., Neupert, W. M., Newmark, J. S., Delaboudinière, J.-P., St. Cyr, O. C., Stezelberger, S., Dere, K. P., Howard, R. A., \& Michels, D. J. 1999, ApJ, 517, L151

Thompson, B. J., Plunkett, S. P., Gurman, J. B., Newmark, J. S., St. Cyr, O. C., \& Michels, D. J. 1998, Geophys. Res. Lett., 25, 2465 
Uchida, Y. 1968, Sol. Phys., 4, 30

Vršnak, B. 2005, EOS Transactions, 86, 112

Vršnak, B., Warmuth, A., Brajša, R., \& Hanslmeier, A. 2002, A\&A, 394, 299

Vršnak, B., Warmuth, A., Temmer, M., Veronig, A., Magdalenić, J., Hillaris, A., \& Karlický, M. 2006, A\&A, 448, 739

Wang, Y.-M. 2000, ApJ, 543, L89

Warmuth, A., Mann, G., \& Aurass, H. 2005, ApJ, 626, L121

Warmuth, A., Vršnak, B., Aurass, H., \& Hanslmeier, A. 2001, ApJ, 560, L105

Warmuth, A., Vršnak, B., Magdalenić, J., Hanslmeier, A., \& Otruba, W. 2004, A\&A, 418, 1101

Wills-Davey, M. J. \& Thompson, B. J. 1999, Sol. Phys., 190, 467

Wu, S. T., Zheng, H., Wang, S., Thompson, B. J., Plunkett, S. P., Zhao, X. P., \& Dryer, M. 2001, J. Geophys. Res., 106, 25089

Young, P. R. \& Esser, R. 1999, Space Science Reviews, 87, 345 

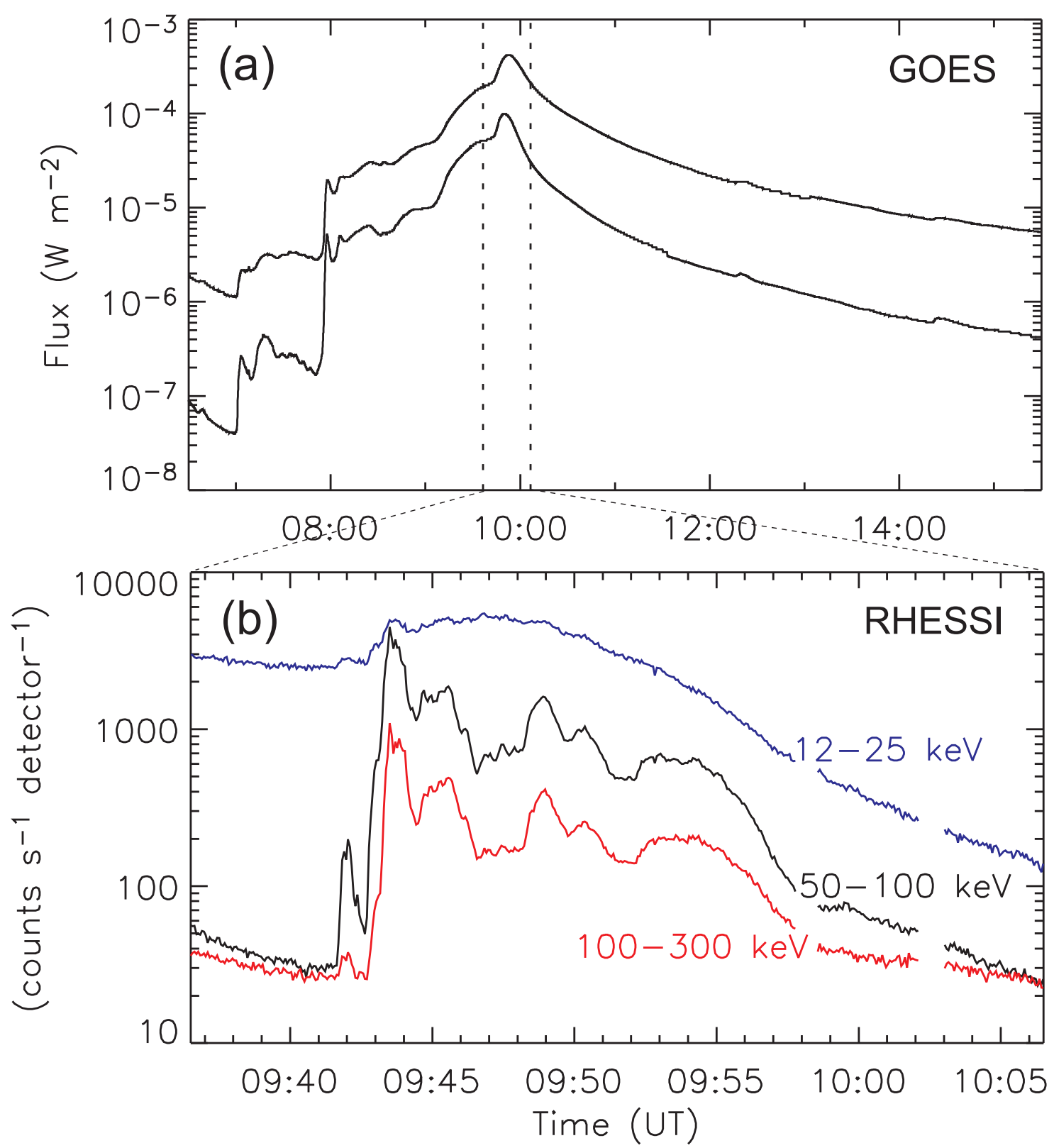

Fig. 1.- a): GOES 0.5-4 and 1-8 A flux. b) RHESSI light curves in the 12-25, 50-100, and 100-300 keV energy bands (4-s integration). [See the electronic edition of the Journal for a color version of this figure.] 


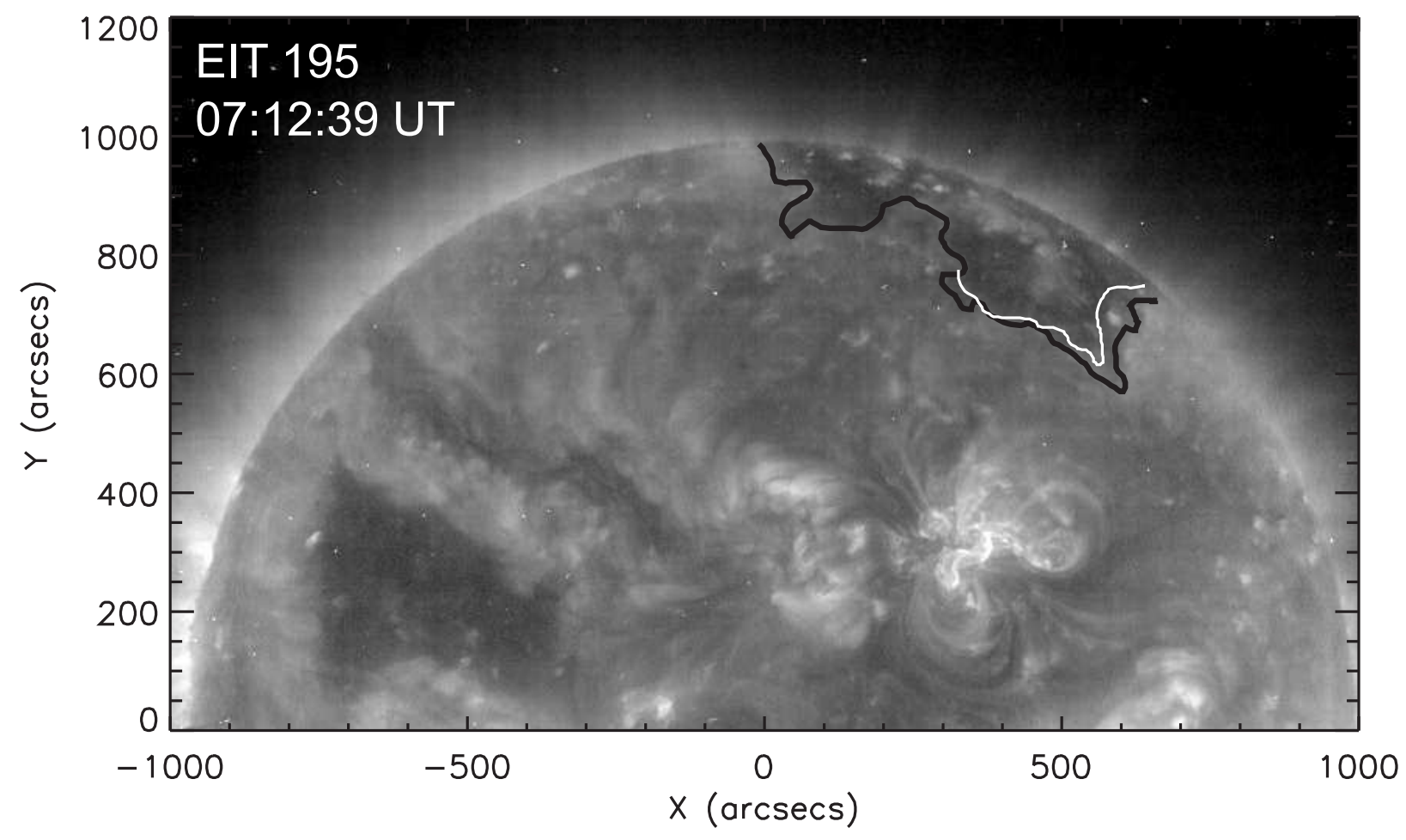

Fig. 2.- EIT $195 \AA$ image of the northern hemisphere of the Sun. The polar CH boundaries are outlined. The black line indicates the outer, the white line the inner estimate of the CH boundary. 

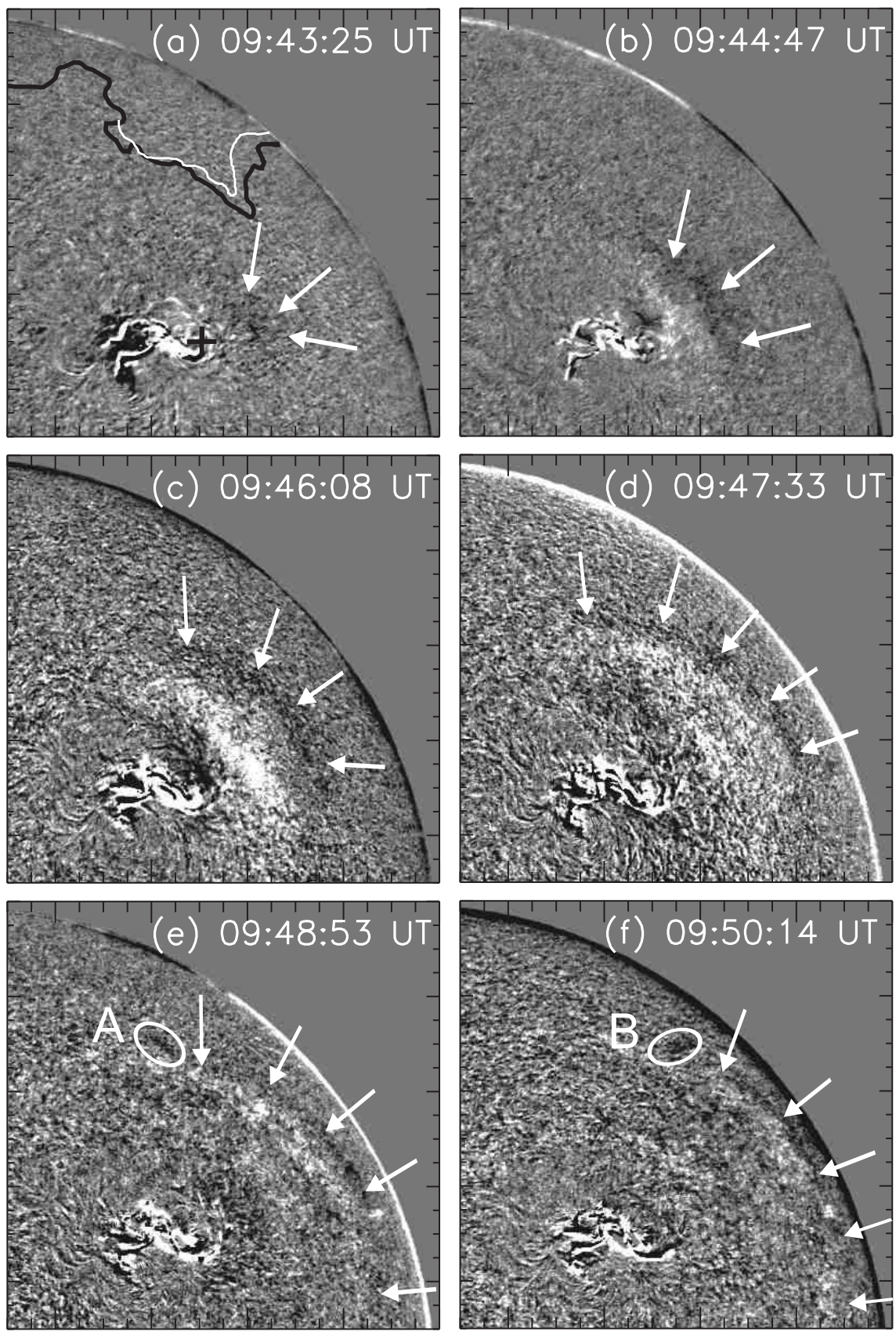

Fig. 3.- Sequence of $\mathrm{H} \alpha+0.4 \AA$ running difference images. On both axes, the plotted field of view extends from $100^{\prime \prime}$ to $1000^{\prime \prime}$ from Sun center. The leading edges of the Moreton wave fronts (seen as dark fronts in $\mathrm{H} \alpha$ red wing observations) are indicated by arrows. In panel a, the determined wave ignition center is indicated by a cross; the $\mathrm{CH}$ boundaries are marked by white and black lines for the inner and outer estimates, respectively. In panels e and $\mathrm{f}$, activated features are indicated as "A" and "B", respectively. [See the electronic edition of the Journal for a color version of this figure.] 


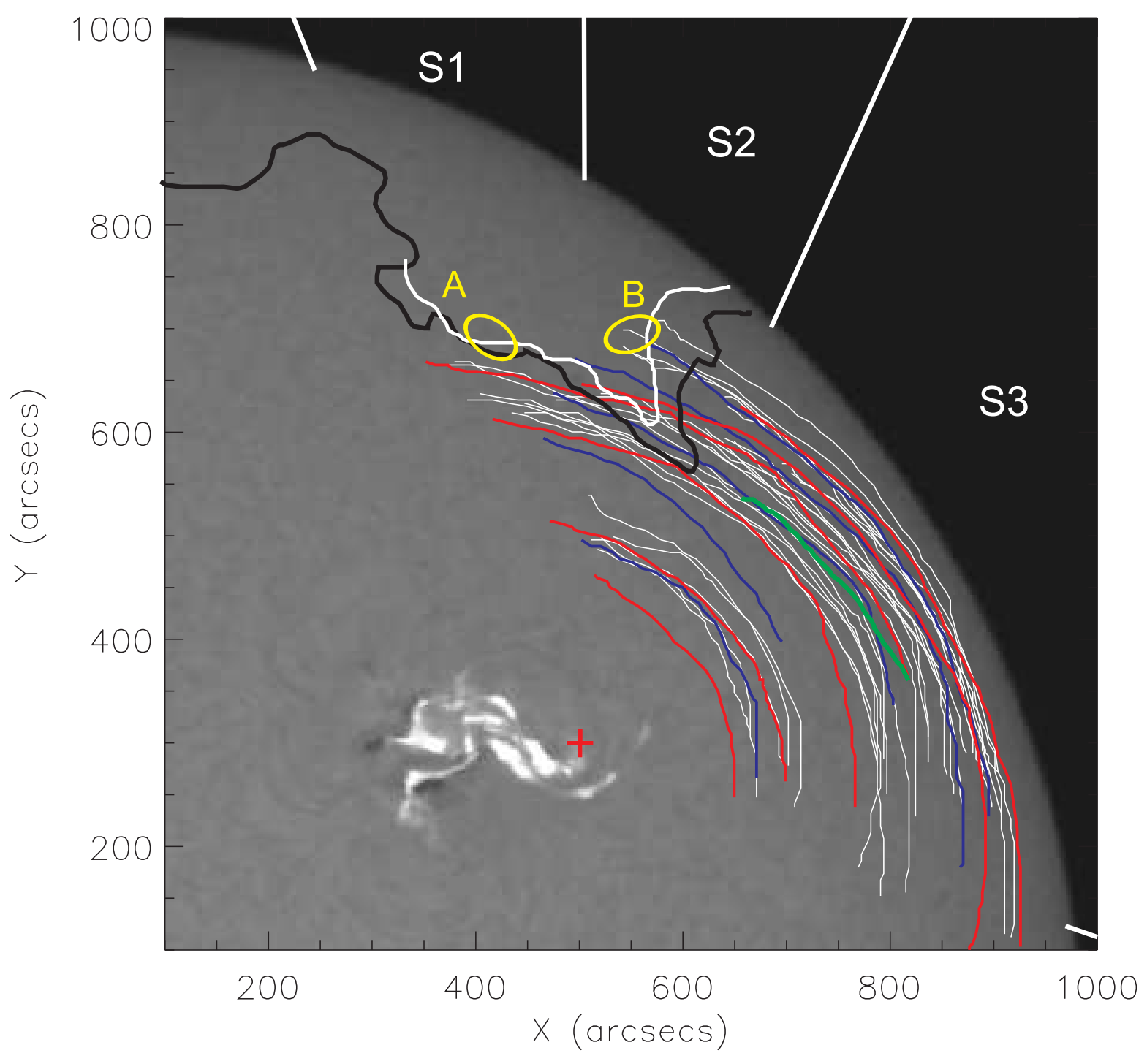

Fig. 4.- Moreton wave fronts visually determined from $\mathrm{H} \alpha$ (white lines), $\mathrm{H} \alpha+0.4 \AA$ (red lines) and $\mathrm{H} \alpha-0.3 \AA$ (blue lines) filtergrams plotted on an $\mathrm{H} \alpha+0.4 \AA$ flare image acquired at 09:47:33 UT. The EIT wave front is plotted in green color. The polar $\mathrm{CH}$ boundaries derived from the EIT image in Fig. 2 are also indicated. The cross marks the determined ignition center of the wave. The yellow ellipses indicate the activated features "A" and "B". The three propagation sectors considered in the kinematical study are indicated by straight lines radial to the wave ignition center; the sectors are denoted as "S1", "S2" and "S3". 

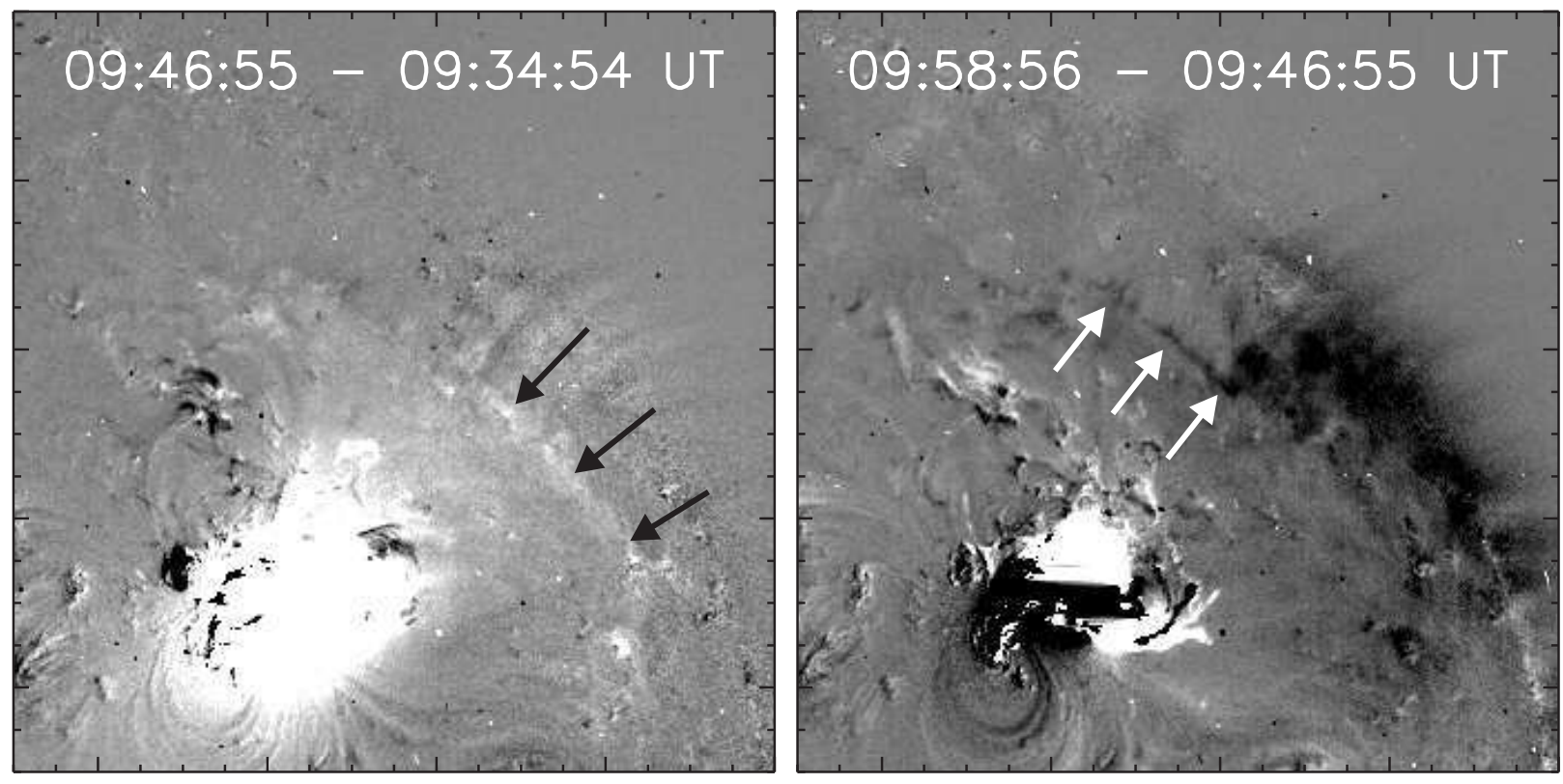

Fig. 5.- EIT $195 \AA$ Å running difference images. The left panel shows the EIT wave front (indicated by black arrows). The right panel reveals the disappearance of a bright feature along the $\mathrm{CH}$ boundary (indicated by white arrows) as well as a coronal dimming in the region passed by the wave. On both axes, the plotted field of view extends from $100^{\prime \prime}$ to $1000^{\prime \prime}$ from Sun center. 


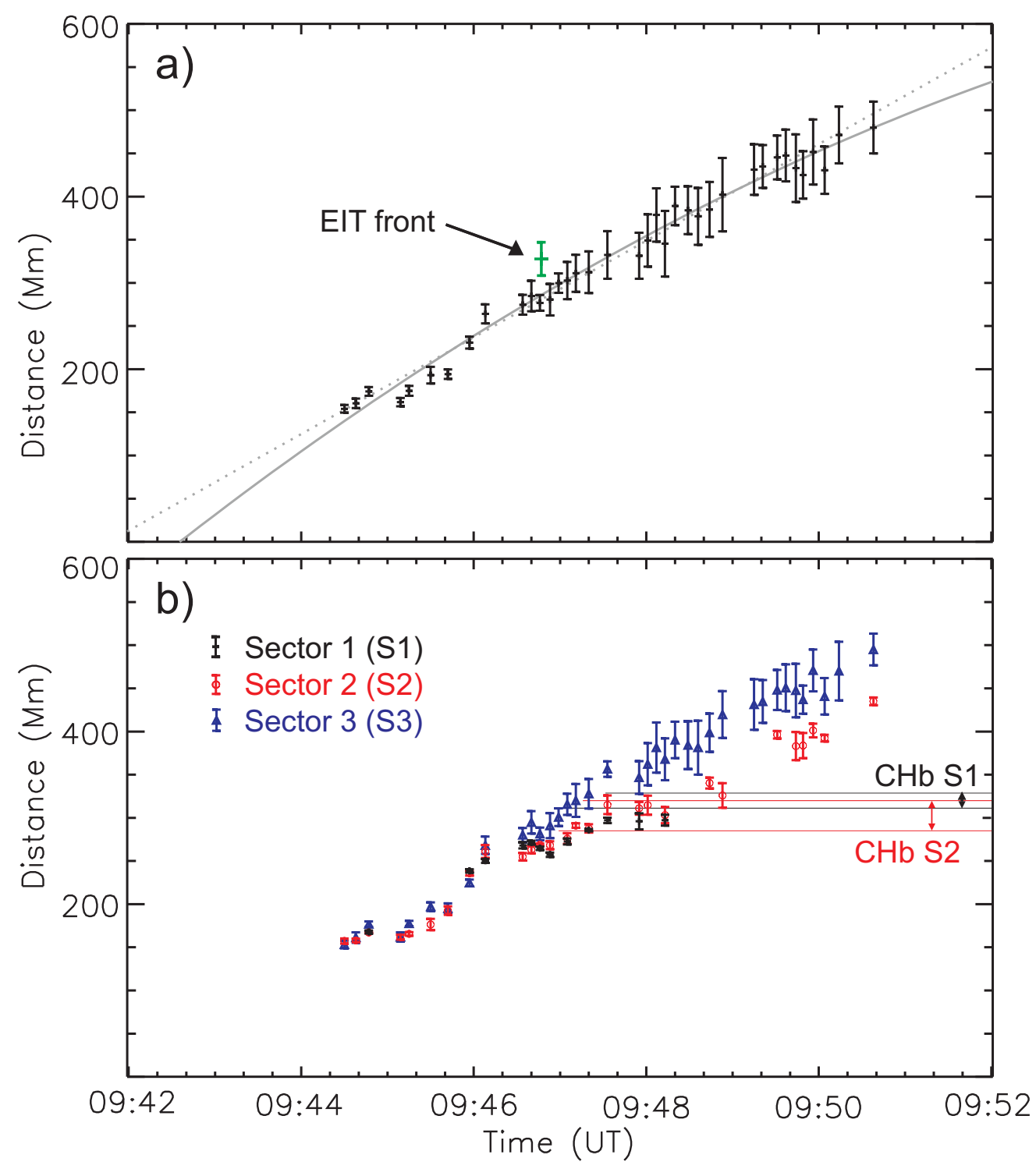

Fig. 6.- Kinematics of the Moreton wave. a) Distance vs. time diagram derived from all points on the measured $\mathrm{H} \alpha$ wave fronts as well as from the EIT wave front. The dotted and solid lines indicate the linear and quadratic fit to the $\mathrm{H} \alpha$ data, respectively. b) Distance vs. time diagram derived separately for three different propagation sectors of the Moreton wave: in the direction of the coronal hole ( $\mathrm{S} 1$, black), in the direction of the $\mathrm{CH}$ tongue ( $\mathrm{S} 2$, red) and in the direction undisturbed by the $\mathrm{CH}$ (S3, blue). The distance of the $\mathrm{CH}$ boundaries into sector S1 ("CHb S1") and towards the tongue in sector S2 ("CHb S2") are indicated by the horizontal lines (lower lines - outer estimates, upper lines - inner estimates). 